Published in final edited form as:

Lancet Infect Dis. 2014 June ; 14(6): 449-450. doi:10.1016/S1473-3099(14)70777-7.

\title{
Artemisinin resistance in Plasmodium falciparum
}

\section{Chanaki Amaratunga, Ph.D.,}

Laboratory of Malaria and Vector Research, National Institute of Allergy and Infectious Diseases, National Institutes of Health, Bethesda, MD, USA

Benoit Witkowski, Ph.D.,

Malaria Molecular Epidemiology Unit, Institut Pasteur du Cambodge, Phnom Penh, Cambodia

Nimol Khim, M.Sc.,

Malaria Molecular Epidemiology Unit, Institut Pasteur du Cambodge, Phnom Penh, Cambodia

Didier Menard, Ph.D., and

Malaria Molecular Epidemiology Unit, Institut Pasteur du Cambodge, Phnom Penh, Cambodia

Rick M. Fairhurst, M.D., Ph.D.*

Laboratory of Malaria and Vector Research, National Institute of Allergy and Infectious Diseases, National Institutes of Health, Bethesda, MD, USA

Artemisinin resistance in Plasmodium falciparum is observed in young (0-3 h) ring-stage parasites, which have a high parasite survival rate in the in-vitro ring-stage survival assay $\left(\mathrm{RSA}^{0-3 \mathrm{~h}}\right.$ ), but then become susceptible as they mature to late rings in the $\mathrm{RSA}^{9-12 \mathrm{~h}}$ and early trophozoites in the TSA ${ }^{18-21 h}$. In the RSA ${ }^{0-3 h}$, young ring-stage parasites are exposed to a pharmacologically relevant dose of dihydroartemisinin for $6 \mathrm{~h}$ and parasite survival is quantified $66 \mathrm{~h}$ later. Using this assay, we recently reported that parasite survival rates differ significantly between fast- and slow-clearing parasite isolates from malaria patients treated with an artemisinin in western Cambodia ( $10.88 \%$ vs $0.23 \%, \mathrm{p}=0.007$, Mann-Whitney test $).{ }^{1}$

Of the 26 parasites tested, however, we found that 4 gave 'discordant' results (Fig 1). Of the 13 fast-clearing parasites, 3 had high survival rates (5.30, 19.32, and 51.39\%) but a resistant stage-dependent pattern $(\Delta=1.2 \%, 17.3 \%$, and $50.2 \%$, respectively). We hypothesized that these 3 parasites were indeed artemisinin-resistant, but that their clearance was fast because circulating parasites seen in blood films were predominantly older, drug-sensitive rings at the time the patients received artemisinins. Of the 13 slow-clearing parasites, 1 had a paradoxically low survival rate $(0.16 \%)$. We hypothesized that this parasite was in fact artemisinin-sensitive, but that its clearance was slow because the patient had low levels of parasite-clearing immunity ${ }^{2}$ or a poor pharmacokinetics profile.

We recently reported K13-propeller polymorphism as a new molecular marker for artemisinin-resistant $P$. falciparum malaria, ${ }^{3}$ and hypothesized that K13-propeller genotypes

"Corresponding author: Rick M. Fairhurst, M.D., Ph.D., Laboratory of Malaria and Vector Research, NIAID/National Institutes of Health, 12735 Twinbrook Parkway, Room 3E-10A, Rockville, MD 20852, USA, Phone: 301-402-7393, Fax: 301-402-2201, rfairhurst@niaid.nih.gov.

We declare that we have no conflicts of interest. 
would definitively resolve the 4 discordant results. To test this possibility we genotyped all 26 parasites and found that the 3 parasites with discordantly high survival rates each carry a different mutant K13-propeller allele (Y493H, C580Y, and $\mathrm{R} 539 \mathrm{H}$ ), and that the parasite with a discordantly low survival rate carries a wild-type allele (Fig 1). Furthermore, all the fast- and slow-clearing parasites with concordant survival rates carry wild-type or mutant alleles (Y493H and C580Y), respectively.

These data suggest that parasite survival rates in the $\mathrm{RSA}^{0-3 \mathrm{~h}}$ are more relevant than parasite clearance half-lives in identifying artemisinin-resistant $P$. falciparum, and further validate K13-propeller polymorphism as a molecular marker of artemisinin resistance in vitro and in vivo.

\section{Acknowledgments}

This work was funded by the Intramural Research Program of the NIAID, NIH and Institut Pasteur du Cambodge (ACIP A14/2012).

\section{References}

1. Witkowski B, Amaratunga C, Khim N, Sreng S, Chim P, Kim S, et al. Novel phenotypic assays for the detection of artemisinin-resistant Plasmodium falciparum malaria in Cambodia: in-vitro and exvivo drug-response studies. The Lancet infectious diseases. 2013; 13(12):1043-9. [PubMed: 24035558]

2. Lopera-Mesa TM, Doumbia S, Chiang S, Zeituni AE, Konate DS, Doumbouya M, et al. Plasmodium falciparum clearance rates in response to artesunate in Malian children with malaria: effect of acquired immunity. J Infect Dis. 2013; 207(11):1655-63. [PubMed: 23448727]

3. Ariey F, Witkowski B, Amaratunga C, Beghain J, Langlois AC, Khim N, et al. A molecular marker of artemisinin-resistant Plasmodium falciparum malaria. Nature. 2014; 505(7481):50-5. [PubMed: 24352242] 


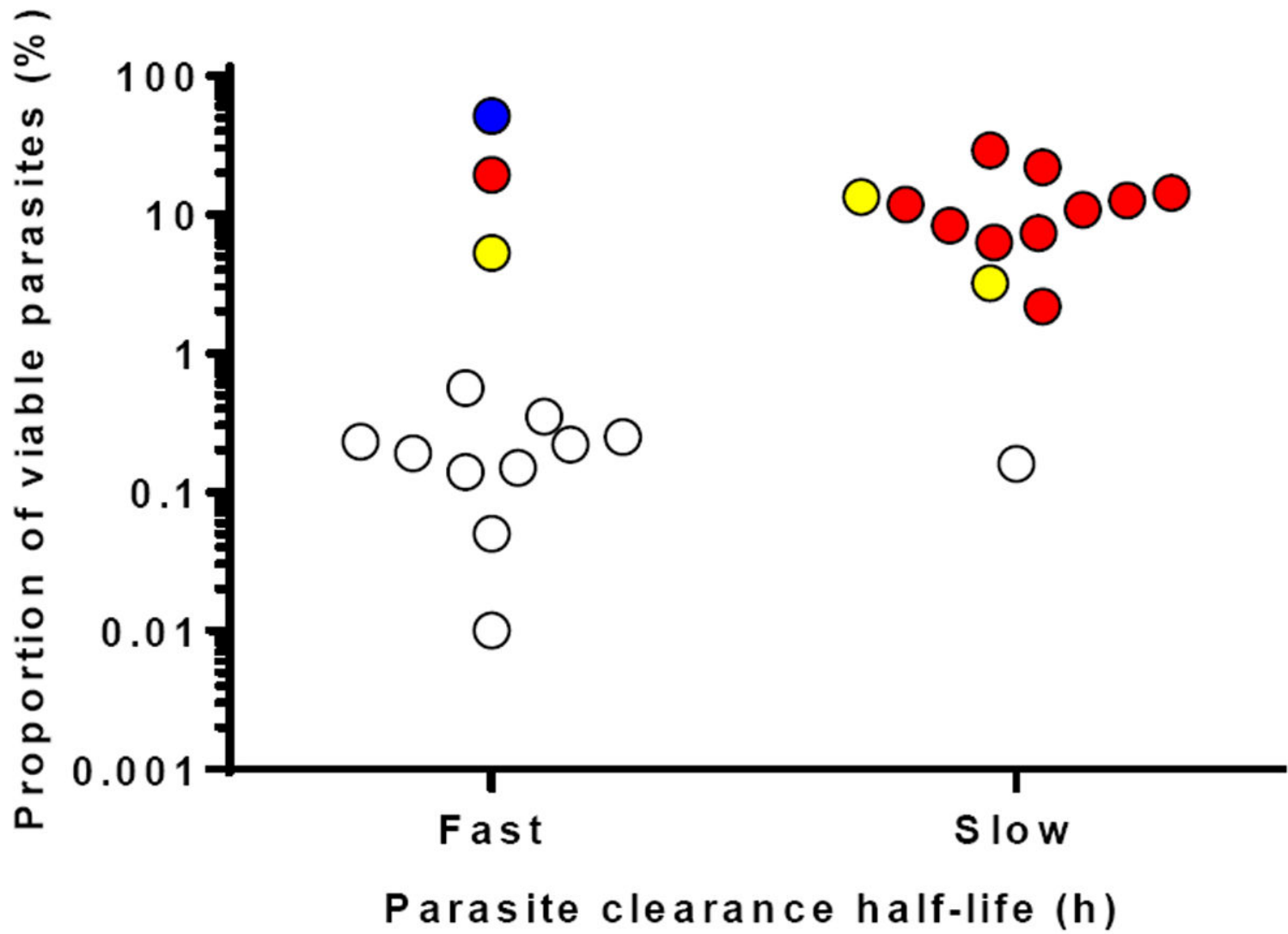

Figure 1.

Associations between parasite survival rates in the in-vitro $\mathrm{RSA}^{0-3 \mathrm{~h}}$, parasite clearance halflives in patients treated with artemisinin-based combination therapy, and K13-propeller alleles: wild-type (clear), R539T (blue), C580Y (red), and Y493H (yellow). 